\title{
Analysis of a $\delta$ Scuti spectroscopic binary: CQ Lyncis ${ }^{\star}$
}

\author{
F. Carrier ${ }^{1}$, Y. Debernardi ${ }^{2}$, S. Udry ${ }^{1}$, J.-M. Carquillat ${ }^{3}$, and N. Ginestet ${ }^{3}$ \\ 1 Observatoire de Genève, 51 chemin des Maillettes, 1290 Sauverny, Switzerland \\ 2 Institut d'Astronomie de l'Université de Lausanne, 1290 Chavannes-des-Bois, Switzerland \\ 3 Observatoire Midi-Pyrénées, Laboratoire d'Astrophysique, 14 Avenue Édouard Belin, 31400 Toulouse, France
}

Received 2 April 2002 / Accepted 13 May 2002

\begin{abstract}
CQ Lyn is a new double-lined spectroscopic binary, composed of an Am $\delta$ Scuti and a solar-like star, with an orbital period of $12.50736 \mathrm{~d}$ and a zero-eccentricity. Physical parameters of both stars are well constrained. The characteristics of the primary are the following: $M=1.714 M_{\odot}, T_{\text {eff }}=6760 \mathrm{~K}, \log L / L_{\odot}=1.097$ and $\log g=3.85$ dex. Power spectra of HIPPARCos photometry and CORAVEL radial velocities show multi-period pulsation behaviour. Three frequencies are identified: $f_{1}=8.86725, f_{2}=8.81150$ and $f_{3}=8.91007 \mathrm{c} / \mathrm{d}$ and are assumed to be $l=2 f$-modes split by rotation, considering their pulsation constants $Q_{1}=0.0356, Q_{2}=0.0359$ and $Q_{3}=0.0355 \mathrm{~d}$. Two combination oscillations are also present in the power spectrum at frequency $f_{1}+f_{2}$ and $f_{2}+f_{3}$.
\end{abstract}

Key words. stars: variables: $\delta$ Sct - stars: binaries: spectroscopic - stars: individual: CQ Lyn

\section{Introduction}

CQ Lyn (HD 67911, HIP 40230) is classified as an $\mathrm{Am}(\mathrm{F} 0 / \mathrm{F} 5 / \mathrm{F} 4)$ star by Abt (1984) and belongs to the red edge of the instability strip; indeed, it was detected as a $\delta$ Scuti type variable star from Hipparcos photometry (ESA 1997; Koen 2001). Such variables are pulsating stars of short period $(<0.3 \mathrm{~d})$ located in the lower part of the Cepheid instability strip, with luminosities ranging from the zero-age-mainsequence (ZAMS) to about $2 \mathrm{mag}$ above the main sequence, with spectral types ranging from about A2 to F2. They pulsate with a large number of excited radial and non-radial modes. Thus, CQ Lyn is an interesting case to investigate the metallicity vs pulsation problem of A-type stars. However, due to the observations of the integrated light across the stellar surface, only low-degree modes can be detected.

Since the early 70s classical Am stars have been found to be stable. Kurtz and his collaborators have investigated the variable Am stars (Kurtz 1978; Kurtz et al. 2000). They concluded that most of $\delta$ Scuti metallic stars are fairly evolved Am stars. Due to Kurtz's (1989) discovery of a classical Am star with $\delta$ Scuti pulsations, the theory of metallicity and pulsation was revised by Turcotte et al. (2000). They concluded that young Am

Send offprint requests to: F. Carrier, e-mail: Fabien.Carrier@obs.unige.ch

* Based on observations collected at the 1.93-m and Swiss 1-m telescopes at the Haute-Provence Observatory (OHP, France), at the Belgian 1.2-m telescope at La Palma (ENO, Spain) and on data from the ESA HiPPARcos satellite. stars are stable against the driving from the $\kappa$ mechanism and that, as the stars evolve, they become unstable near the red edge of the instability strip.

CQ Lyn, which exhibits variations according to the HIPPARCos photometric measurements, was monitored by using the CoRAvel radial velocity scanner. The results of this photometric and radial velocity analysis are presented in this paper.

\section{Observations and reduction}

The observations were obtained with the CORAVEL radial velocity scanner (Baranne et al. 1979) installed on the Swiss 1-m telescope at the Haute-Provence Observatory (OHP) during several observing runs. The radial velocities are related to the Elodie system (Udry et al. 1999). Most of the observations were obtained between 1997 and 1998. Previous measurements were obtained from 1992.

The observations of CQ Lyn started in 1992 with the goal of determining the Am stars' binarity. After the HIPPARcos publication, when CQ Lyn was determined as $\delta$ Scuti, the observational strategy was to devote some nights to follow the short time variations. Overall, 586 radial velocities were obtained with CORAVEL.

Two additional observations were realized to search for a secondary correlation dip in 2002 with the EloDIE spectrograph installed on the 1.93-m telescope at the OHP (Baranne et al. 1996).

In February 2002, this star was measured in the Geneva photometric system (Golay 1980) with the photoelectric 


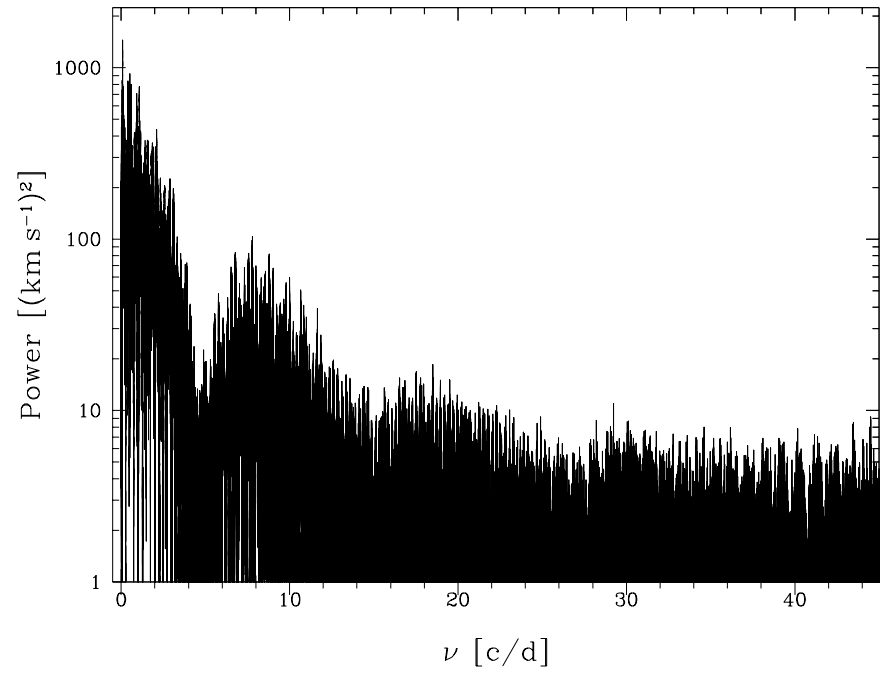

Fig. 1. Power spectrum of the radial velocity measurements of CQ Lyn. Stellar signal can be seen near $0 \mathrm{c} / \mathrm{d}$ (duplicity), 9 and $18 \mathrm{c} / \mathrm{d}$ (pulsations).

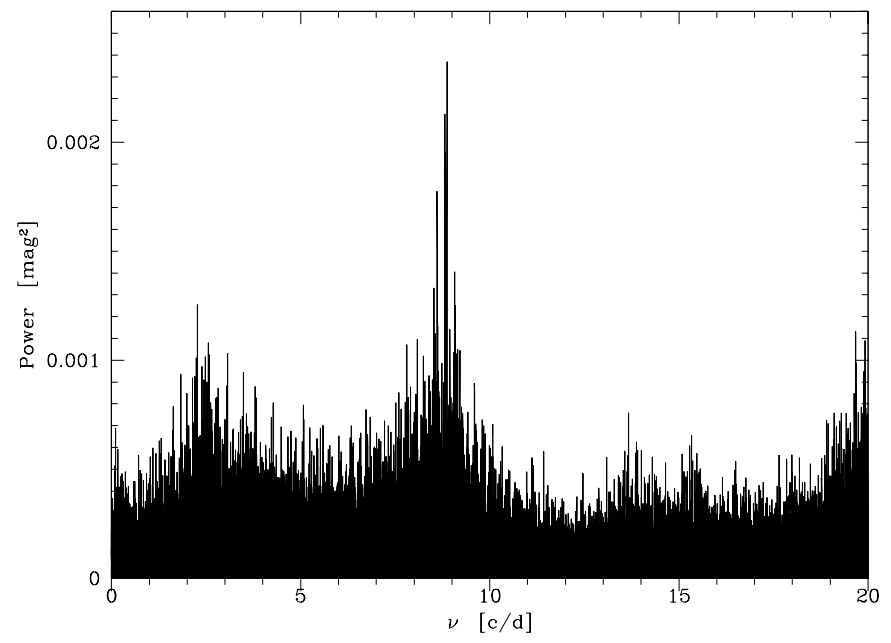

Fig. 2. Power spectrum of the Hipparcos photometry of CQ Lyn. Stellar signal can be seen at $9 \mathrm{c} / \mathrm{d}$ (pulsations).

photometer P7 (Burnet \& Rufener 1979) installed on the 120-cm Belgian telescope in La Palma (ENO, Spain). The photometric reduction procedure is described by Rufener (1964, 1985); the photometric data in the Geneva system are collected in the General Catalogue (Rufener 1988) and its up-todate database (Burki et al. 2002). The measurements are listed in Table 1. In addition to these data, 102 photometric measurements have been obtained by the HiPPARcos satellite (ESA 1997) in the range 7972-8763 (in HJD-2 440000 ).

\section{Variability}

CQ Lyn was detected as a variable star of $\delta$ Scuti type by Hipparcos (ESA 1997). In order to compute the power spectrum of the velocity time series, the Lomb-Scargle modified algorithm was used (Lomb 1976; Scargle 1982) with a weight being assigned to each point according to its uncertainty estimate. The time scale gives a resolution of $0.0005 \mathrm{c} / \mathrm{d}$. Figure 1 shows this periodogram. The Coravel data confirm the $\delta$ Scuti characteristics. Indeed, the power spectrum presents an excess in the range $5-15 \mathrm{c} / \mathrm{d}$ with several significant peaks centred at $9 \mathrm{c} / \mathrm{d}$ degraded by the complicated window function. This excess is clearly a signature of pulsations.

Toward the lowest frequencies, the power should rise and scale inversely with frequency squared as expected for instrumental instabilities. However, the increase is too abrupt: there is a significant frequency at $0.079953 \mathrm{c} / \mathrm{d}$. This periodicity cannot be due to pulsation, because the amplitude is too large $\left(40 \mathrm{~km} \mathrm{~s}^{-1}\right)$ and the frequency is moreover not detected through the HIPPARcos photometry (see Fig. 2). To verify the binarity hypothesis, CQ Lyn was observed with the spectrograph ELODIE. A secondary correlation dip is detected: CQ Lyn is in fact a double-lined binary with an orbital frequency of $0.079953 \mathrm{c} / \mathrm{d}(P=12.50736 \mathrm{~d})$.

A preliminary fit of the orbital solution determined an eccentricity of 0.02. Thus, the test of Lucy \& Sweeney (1971) was applied in order to see whether this small eccentricity is significant or not. The probability $p$ is equal to 0.371 in our case, which is much greater than the limit of 0.05 determined by Lucy \& Sweeney. Therefore this eccentricity of 0.02 is not significant and is fixed to zero. As the orbital motion also follows a sinusoidal curve, the orbital and pulsation frequencies can be determined with the same algorithm (see Sect. 4).

\section{Frequency analysis}

One of the most important questions in the examination of multiperiodicity concerns the decision that a detected peak in the power spectrum can be regarded as intrinsic to the star. Only the peaks with a ratio of amplitude signal/noise $>4$ have been considered (Breger et al. 1995). For the detection of frequency combinations, the criterion was relaxed to 3 , since the frequency values during the search are already known.

To resolve the pulsations of CQ Lyn, each real frequency in the power spectrum is extracted step by step. The sinusoidal variation corresponding to the previously identified frequencies is subtracted from the original time series and the power spectrum is calculated again. This operation is repeated until there is no more frequency above the 4- $\sigma$ level. Finally, a multiple linear least square fitting method is used to determine the frequency amplitudes.

The power spectrum of the two independent sets of measurements, Hipparcos photometry and CORAVEl radial velocities, are calculated. The first cleaned frequency in the CoRAVEL power spectrum is the orbital one $(0.079953 \mathrm{c} / \mathrm{d})$ : this frequency does not appear in the HIPPARcos photometry. Although the photometric power spectrum (see Fig. 2) has a resolution of $13 \times 10^{-4} \mathrm{c} / \mathrm{d}$ and has a lot of noise, it can help select the real frequencies in the radial velocity power spectrum, which has a resolution of $5 \times 10^{-4} \mathrm{c} / \mathrm{d}$ and a noise, estimated in the range $45-65 \mathrm{c} / \mathrm{d}$, of $0.15 \mathrm{~km} \mathrm{~s}^{-1}$. Indeed, the Coravel window function (Fig. 3) shows several aliases. Therefore, combining both power spectra allows us to unambiguously determine the 8.86725 and $8.81150 \mathrm{c} / \mathrm{d}$ frequencies (see Fig. 4). A third frequency $8.91007 \mathrm{c} / \mathrm{d}$, with shorter amplitude, is detected only in the radial velocity time series. As seen in Fig. 3, peaks looking like the window function are always present: the signal still 
Table 1. Geneva photometric data of CQ Lyn. $Q$ and $P$ are the individual weights for the magnitude and the colours, varying from 0 to 4 . The column PJ is HJD - 2452 317. The last row is the mean value corrected from the pulsation.

\begin{tabular}{|c|c|c|c|c|c|c|c|c|c|}
\hline$P J$ & $Q$ & $V$ & $P$ & {$[U-B]$} & {$[V-B]$} & {$[B 1-B]$} & {$[B 2-B]$} & {$[V 1-B]$} & {$[G-B]$} \\
\hline 0.446 & 2 & 7.959 & 3 & 1.511 & 0.514 & 0.983 & 1.369 & 1.228 & 1.598 \\
\hline 0.458 & 2 & 8.021 & 3 & 1.517 & 0.493 & 0.996 & 1.374 & 1.211 & 1.583 \\
\hline 0.474 & 2 & 8.037 & 3 & 1.495 & 0.474 & 0.998 & 1.376 & 1.197 & 1.558 \\
\hline 0.488 & 2 & 8.067 & 3 & 1.490 & 0.466 & 1.001 & 1.362 & 1.191 & 1.546 \\
\hline 0.509 & 2 & 8.036 & 3 & 1.480 & 0.478 & 0.998 & 1.362 & 1.195 & 1.562 \\
\hline \multirow[t]{2}{*}{0.527} & 2 & 7.962 & 3 & 1.497 & 0.510 & 0.987 & 1.379 & 1.227 & 1.601 \\
\hline & & 8.006 & & 1.498 & 0.493 & 0.993 & 1.371 & 1.211 & 1.579 \\
\hline
\end{tabular}

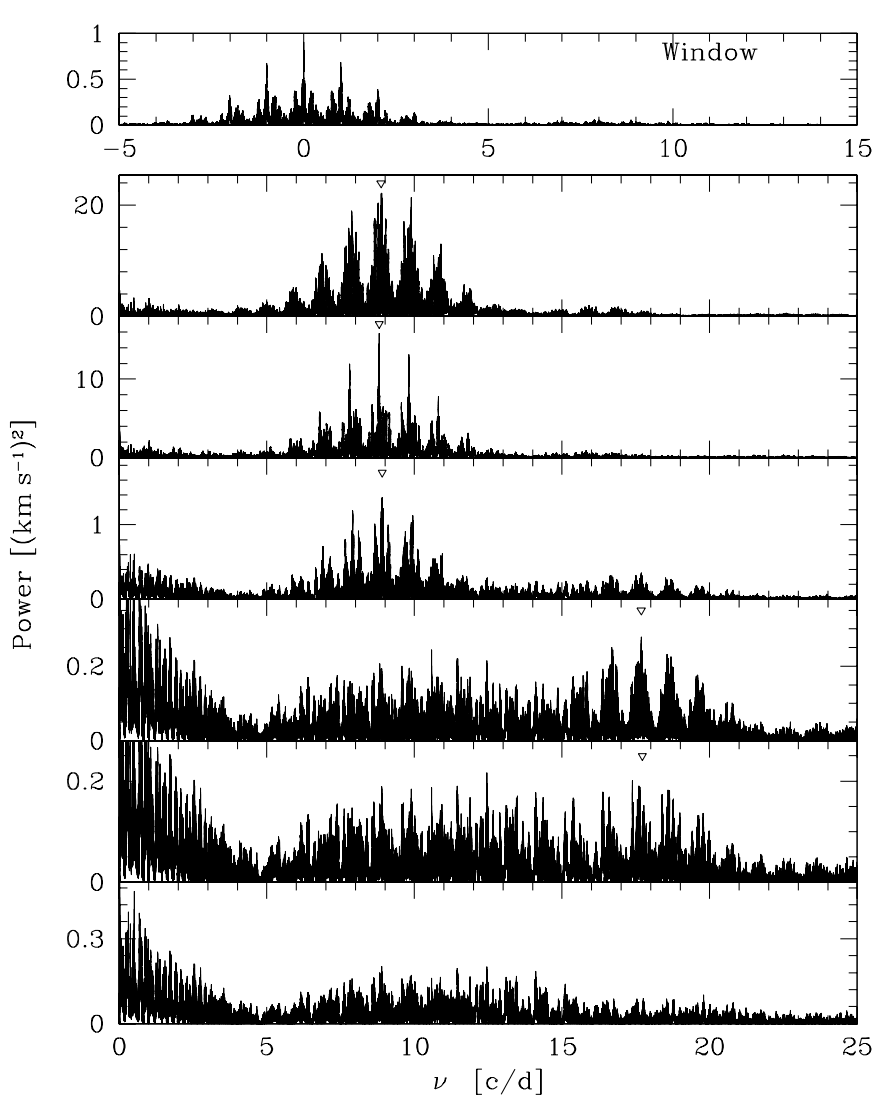

Fig. 3. Power spectrum of the radial velocity measurements of CQ Lyn. The spectral window is shown in the top panel. Five frequencies can be identified (open triangles) in the next five panels. The bottom panel shows the residuals after cleaning all five frequencies. The orbital frequency $(0.079953 \mathrm{c} / \mathrm{d})$ was already cleaned.

appears, near $18 \mathrm{c} / \mathrm{d}$. Moreover, it exactly corresponds to the combination of the three previously determined frequencies. $f_{4}$ and $f_{5}, 17.67875$ and $17.72156 \mathrm{c} / \mathrm{d}$, respectively correspond to $f_{1}+f_{2}$ and $f_{2}+f_{3}$. After removal of the five pulsation modes, the residuals (see bottom panel of Fig. 3) only show a small excess between 5 and $15 \mathrm{c} / \mathrm{d}$, indicating either some non-detected modes or perhaps inadequate removal of the signal. The frequencies are listed in Table 2 with their estimated amplitudes.

\section{Orbital solution}

The orbital period is fitted and described in Sects. 3 and 4. The period is $12.50736 \mathrm{~d}$ and the eccentricity is fixed to 0 .

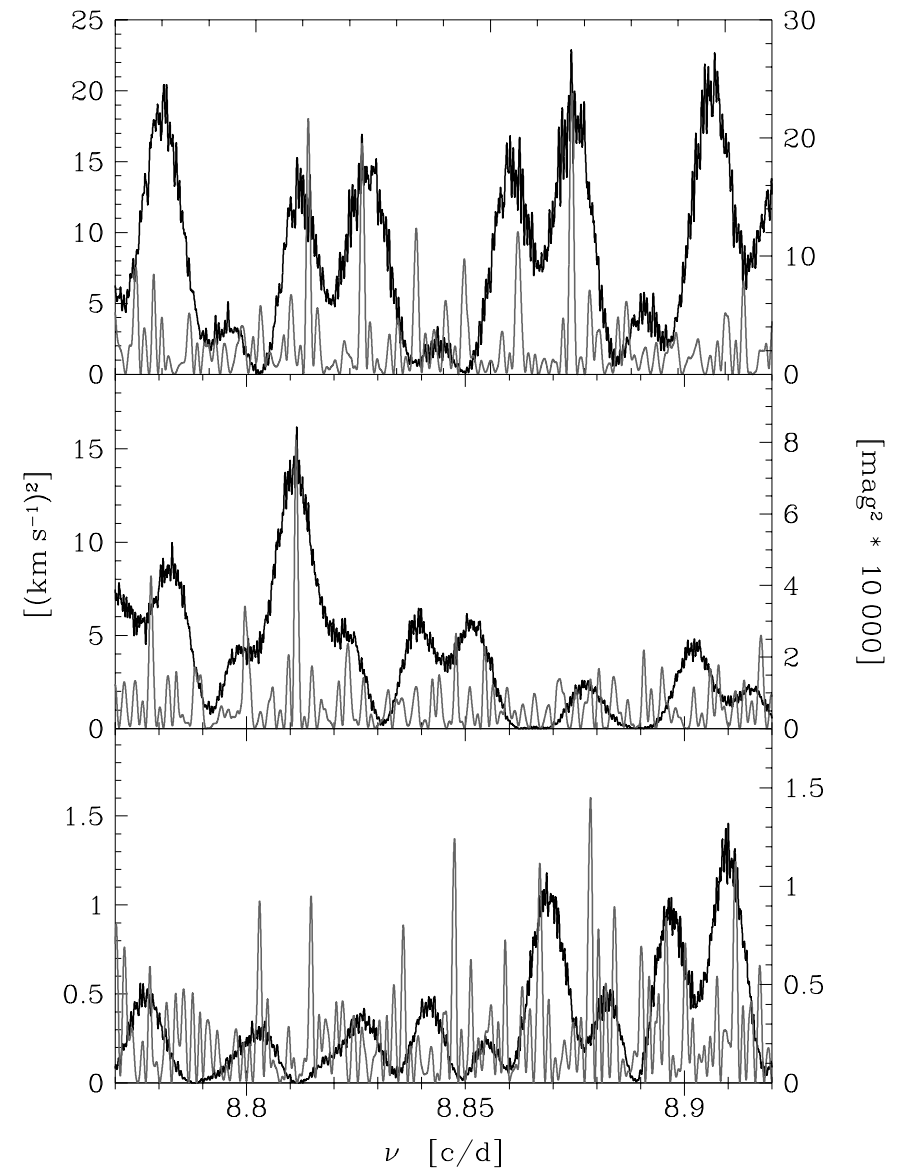

Fig. 4. Power spectrum of the radial velocity measurements (black) and of the HIPPARcos photometry (gray) of CQ Lyn. These two independent sets of measurements allow us to unambiguously determine the frequencies 8.86725 and $8.81150 \mathrm{c} / \mathrm{d}$. The first panel shows the initial Fourier transform (cleaned of the duplicity frequency in the radial velocities case). In the second panel, only the $8.86725 \mathrm{c} / \mathrm{d}$ frequency is cleaned, and in the bottom panel both frequencies are cleaned.

Measurements obtained with the spectrograph ELODIE allowed to see the secondary dip in the cross-correlation function (CCF). Thus, all CORAvel CCF were reinvestigated to search for the secondary. Due to the range of the correlation windows (70 $\mathrm{km} \mathrm{s}^{-1}$ wide), only a tenth of the measurements show the presence of the companion.

In spite of a lack of secondary measurements, the accuracy of the orbital solution allows us to be very confident of the 
Table 2. The frequency spectrum of CQ Lyn. ${ }^{*} f_{0}$ corresponds to the orbital solution.

\begin{tabular}{lrrrr}
\hline \hline & Frequency & \multicolumn{2}{c}{ Amplitude } & $Q$ \\
& $\mathrm{c} \mathrm{d}^{-1}$ & $\mathrm{~km} \mathrm{~s}^{-1}$ & $\mathrm{mag}$ & \multicolumn{1}{c}{$\mathrm{d}$} \\
\hline$f_{0}{ }^{*}$ & 0.07995290 & 40.009 & - & - \\
$f_{1}$ & 8.867252 & 5.183 & 0.040 & $0.0356 \pm 0.0058$ \\
$f_{2}$ & 8.811499 & 4.397 & 0.031 & $0.0359 \pm 0.0059$ \\
$f_{3}$ & 8.910065 & 0.827 & - & $0.0355 \pm 0.0058$ \\
$f_{4}=f_{1}+f_{2}$ & 17.67875 & 0.578 & - & - \\
$f_{5}=f_{2}+f_{3}$ & 17.72156 & 0.428 & - & - \\
\hline
\end{tabular}

Table 3. Orbital solution of CQ Lyn.

\begin{tabular}{lc}
\hline \hline$P[\mathrm{~d}]$ & $12.50736 \pm 0.00008$ \\
$T[\mathrm{HJD}-2440000]$ & $9998.7627 \pm 0.0029$ \\
$e$ & 0 \\
$\gamma\left[\mathrm{km} \mathrm{s}^{-1}\right]$ & $-1.145 \pm 0.037$ \\
$\omega\left[^{\circ}\right]$ & - \\
$K_{1}\left[\mathrm{~km} \mathrm{~s}^{-1}\right]$ & $40.009 \pm 0.067$ \\
$K_{2}\left[\mathrm{~km} \mathrm{~s}^{-1}\right]$ & $63.898 \pm 0.352$ \\
$q=K_{2} / K_{1}$ & $0.6261 \pm 0.0036$ \\
$a_{1} \sin (i)[G m]$ & $6.881 \pm 0.012$ \\
$a_{2} \sin (i)[G m]$ & $10.992 \pm 0.061$ \\
$M_{1} \sin ^{3}(i)\left[M_{\odot}\right]$ & $0.896 \pm 0.009$ \\
$M_{2} \sin ^{3}(i)\left[M_{\odot}\right]$ & $0.561 \pm 0.005$ \\
\hline
\end{tabular}

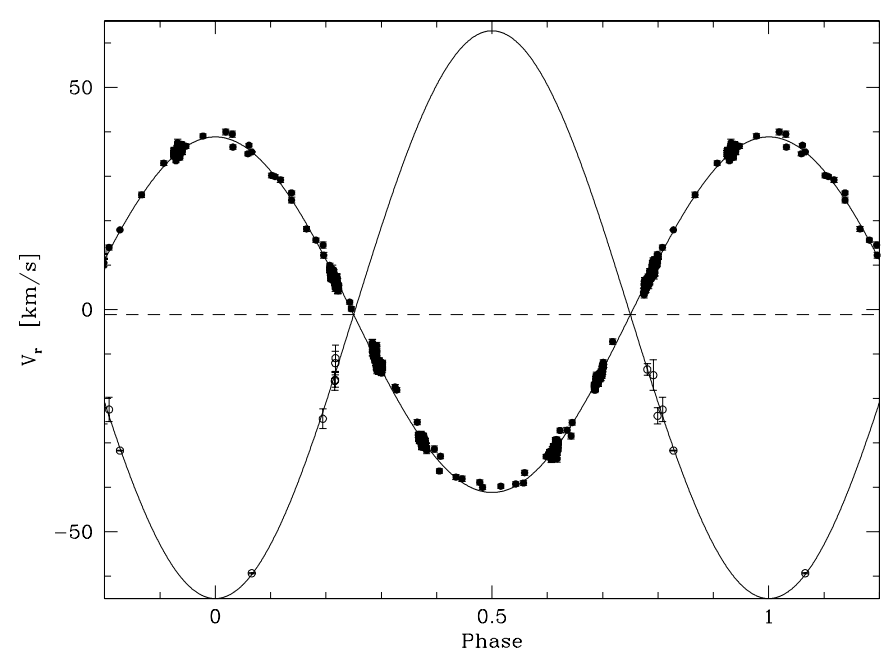

Fig. 5. Radial velocity curve of CQ Lyn. The period is 12.50736 d. All determined pulsation frequencies are cleaned.

determination of the mass ratio $q$. The fitted orbit is plotted in Fig. 5 and the orbital elements are listed in Table 3. The scattering for both components, considering the composition of the orbital motion and the pulsation, is $0.88 \mathrm{~km} \mathrm{~s}^{-1}$.

\section{Physical properties}

Geneva photometric data (Table 1) are used to determine the physical characteristics of CQ Lyn. The main difficulty is to
Table 4. Physical parameters of CQ Lyn.

\begin{tabular}{lc}
\hline \hline Log(Age $)$ & $9.171 \pm 0.036$ \\
$M_{1}\left[M_{\odot}\right]$ & $1.714 \pm 0.061$ \\
$M_{2}\left[M_{\odot}\right]$ & $1.073 \pm 0.038$ \\
$\log L_{1} / L_{\odot}$ & $1.097 \pm 0.085$ \\
$\log L_{2} / L_{\odot}$ & $0.045 \pm 0.078$ \\
$T_{\text {eff1 }}\left[{ }^{\circ} \mathrm{K}\right]$ & $6760 \pm 50$ \\
$T_{\text {eff2 }}\left[{ }^{\circ} \mathrm{K}\right]$ & $5930 \pm 110$ \\
$R_{1}\left[R_{\odot}\right]$ & $2.58 \pm 0.28$ \\
$R_{2}\left[R_{\odot}\right]$ & $1.00 \pm 0.11$ \\
$\log (g)_{1}$ & $3.846 \pm 0.076$ \\
$\log (g)_{2}$ & $4.466 \pm 0.030$ \\
\hline
\end{tabular}

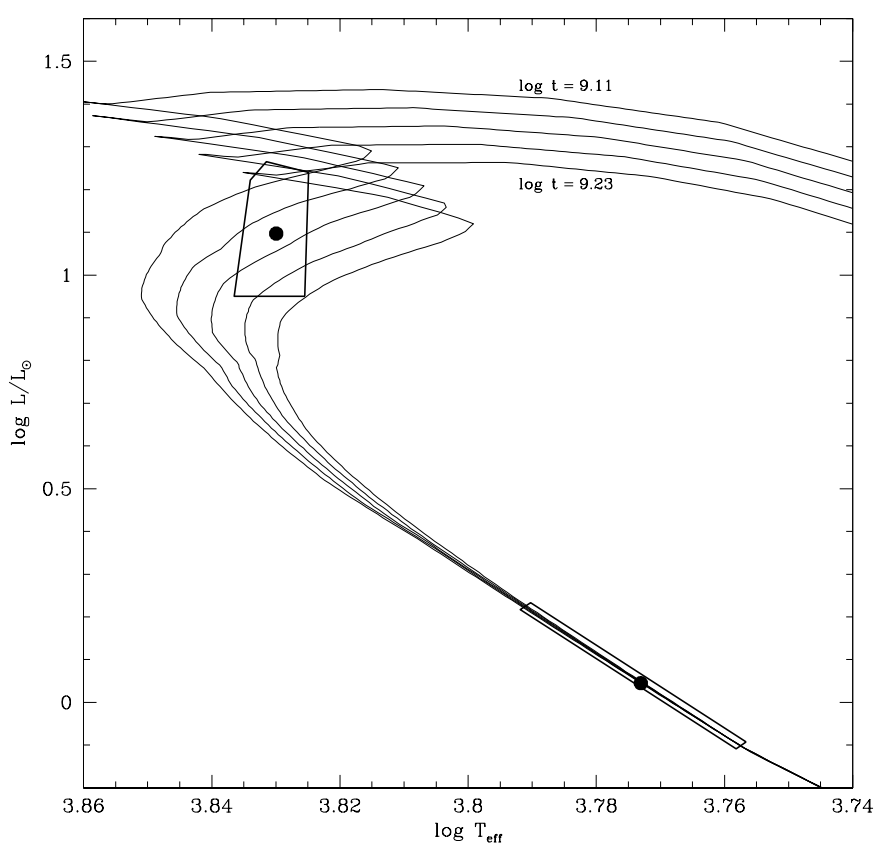

Fig. 6. HR diagram. Both components of CQ Lyn are plotted: the dots represent the mean values and the box surrounds all possible values. The isochrones are traced by step of 0.03 in $\log t$.

disentangle both components. First, the photometric observations are placed in an observational HR diagram $(B-V)$ vs. $M_{V}$, by subtracting the effects due to the reddening and to the distance, taking into account a colour excess $E(B-V)=0.007$ (Lucke 1978) and the Hipparcos parallax $\pi=6.14 \pm 0.99$ mas (ESA 1997). The relations of Meylan \& Hauck (1981) are used to convert the Geneva into Johnson photometries. By using the evolutionary tracks of Schaller et al. (1992) and the mass ratio $q=0.6261$, it is possible to determine, in the observational HR diagram, a couple of stars, on a same isochrone, which correspond to the double system.

The possible solutions are shown in Fig. 6. There are two different cases depending on the evolution of the primary: either it belongs to the main sequence or it has just left the TAMS (terminal-age-main-sequence). This last case is possible but less probable due to the time spent on this stage in an 
Table 5. Rotational velocities of CQ Lyn.

\begin{tabular}{lc}
\hline \hline$V_{A} \sin i\left[\mathrm{~km} \mathrm{~s}^{-1}\right]$ & $11.7 \pm 1.0$ \\
$V_{B} \sin i\left[\mathrm{~km} \mathrm{~s}^{-1}\right]$ & $3.9 \pm 1.0$ \\
$V_{A}\left[\mathrm{~km} \mathrm{~s}^{-1}\right]$ & $12.9 \pm 1.4$ \\
$V_{B}\left[\mathrm{~km} \mathrm{~s}^{-1}\right]$ & $4.8 \pm 1.3$ \\
$V_{\mathrm{sync} A}\left[\mathrm{~km} \mathrm{~s}^{-1}\right]$ & $10.43 \pm 1.13$ \\
$V_{\mathrm{sync} B}\left[\mathrm{~km} \mathrm{~s}^{-1}\right]$ & $4.04 \pm 0.44$ \\
\hline
\end{tabular}

evolutionary track, however, it has to be taken into account. The models give physical parameters listed in Table 4.

The errors are determined from the distribution of possible solution of all parameters explained above. A ponderation, depending on the gap between the considered set of values and the average solution, is applied. The errors on parameters are finally calculated by fitting a Gaussian on each distribution.

\section{Rotational velocities}

The rotational velocity of the primary is determined with the relation using parameters of the CORAVEL correlation peaks (Benz $\&$ Mayor 1984). The Coravel cross-correlation peaks of the secondary do not allow us to determine its rotational velocity, because the peaks are poorly defined. Therefore, a similar relation given for the ELODIE spectrograph is used (Queloz et al. 1998), where the secondary peak is well defined.

The inclination $i$, calculated from the mass $M$ and $M \sin ^{3}(i)$ of the star, is about $54^{\circ}$. Moreover, we can note that the orbit is circularized and synchronized, $V_{\text {sync }}$ being determined from the orbital period and the radii of both components (Table 5).

\section{Pulsation analysis}

The three main pulsation modes $\left(f_{1}\right.$ to $f_{3}$ ), being very close, could be due to aliasing effects or variation in amplitude of only one frequency (Breger \& Bischof 2002). However, as they are detected from two independent data sets (HIPPARcos and CORAVEL), which are not simultaneously obtained, they are real frequencies.

One of the main problems concerning the interpretation of observed frequencies is to identify their pulsation modes. The power spectra of solar-like oscillations show well defined patterns of peaks; in contrast, the spectra of $\delta$ Scuti stars show no regularities on any scale. This complication is due in part to the fast rotation of such stars, but above all only a tiny fraction of the possible modes are excited. The pulsation modes identification can be done either from analysis of profile variations of a high resolution spectral line, or from the phase difference and amplitude ratio of colors variations (see for example Balona et al. 2001). Another method is to compare the theoretical pulsation constant and the observational one. The well-known relation

$P \sqrt{\rho / \rho_{\odot}}=Q$,

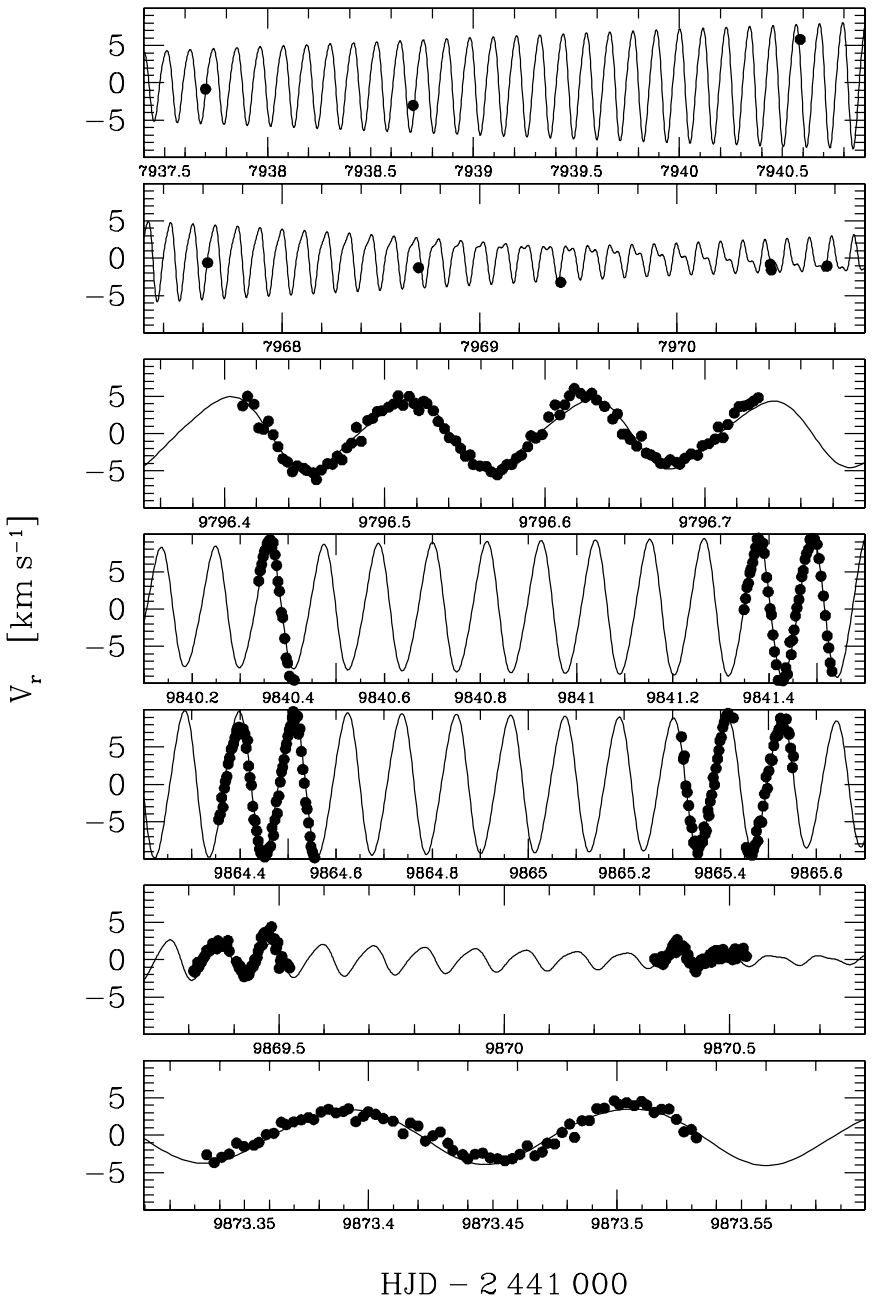

Fig. 7. The fits of the five frequencies to the radial velocity data for some nights (after subtracting the orbital solution).

where $P$ is the pulsation period, $\rho$ the stellar density and $Q$ the pulsation constant, can be written in the form (Breger 2000)

$\begin{aligned} \log Q= & \log P+0.5 \log \left(M / M_{\odot}\right)+0.3 M_{\mathrm{bol}} \\ & +3 \log T_{\mathrm{eff}}-12.7085\end{aligned}$

taking $T_{\text {eff }, \odot}=5770 \mathrm{~K}$ and $M_{\text {bol, } \odot}=4.75$ (Allen 1973; Labs $\&$ Neckel 1970). This constant is determined from the parameters calculated in Sect. 6 and listed in Table 4 and is thus for the three frequencies: $Q_{1}=0.0356 \pm 0.0058, Q_{2}=$ $0.0359 \pm 0.0059$ and $Q_{3}=0.0355 \pm 0.0058 \mathrm{~d}$. Comparing these $Q$-values with the theoretical ones (Fitch 1981; Dziembowski \& Pamyatnykh 1991), all are in perfect agreement with the radial fundamental mode. However, the three frequencies cannot all be radial fundamental modes. The most probable identification is that these frequencies correspond to $f$-modes with $l=2$ $(Q=0.0344$, Fitch 1981). Other identifications within acoustic modes such as $(n=1, l=1)$ and $(n=1, l=2)$-modes cannot be ruled out: $Q$-values for $(n=1, l=1)$ and $(n=1, l=2)$ modes are expected around 0.0287 and $0.0264 \mathrm{~d}$ (Fitch 1981) respectively, only at 1.2 and $1.6 \sigma$. The frequencies of the three modes being very accurate imply that all modes have nearly the same pulsation constant $Q$ and that all three frequencies 
would be the same mode split by rotation. Tidal and/or high order rotational splittings can explain the observed asymmetrical separation between the modes. Thus, the most probable explanations are:

- The three modes are $l=2 f$-modes split by rotation with $\Delta m$ equal to 1 between adjacent frequencies since the rotational frequency is about $0.1 \mathrm{~d}^{-1}$.

- The three modes fall within the range of $g$-mode oscillations

Two combination frequencies were also detected $\left(f_{4}=f_{1}+f_{2}\right.$ and $f_{5}=f_{2}+f_{3}$ ). They both have small amplitudes. However, a one-to-one relationship does not exist between the amplitudes of the combination frequencies and the main frequencies involved in the combinations (Dziembowski 1982).

The pulsations are stable along the time. The same main frequencies were detected in the photometric (1990-1992) and radial velocity (1992-1998) surveys, which were realized at two different epochs. Moreover, the amplitude of the frequencies seems to be constant during the whole radial velocity survey.

\section{Conclusion}

This radial velocity survey was extremely fruitful, since we found that CQ Lyn was a new double-lined spectroscopic binary and that the primary is a $\delta$ Scuti type star with three main frequencies and two combination frequencies. The difference between the main frequencies is very small and a long-term survey (longer than typical missions of one or two weeks) is needed to obtain a sufficient resolution. Moreover, due to its belonging to a binary system, its physical parameters are well constrained. CQ Lyn is another example showing that even for Am stars, there is sufficient residual helium left to drive $\delta$ Scuti pulsation Thus, it is clear that pulsation and metallicism can coexist in low instability strip.

Finally, it would be very important to obtain multi-colour photometry to improve the mode identification.

Acknowledgements. We are grateful to C. Aerts and K. Uytterhoeven for obtaining the Geneva photometry for us, G. Burki for making the reduction and X. Delfosse and D. Naef for the observations with ELODIE spectrograph. This work has been partly supported by the Swiss National Science Foundation.

\section{References}

Abt, H. A. 1984, ApJ, 285, 247

Allen, C. W. 1973, Astrophysical Quantities, 3rd ed. (Athlone Press, London), 162

Balona, L. A., Bartlett, B., Caldwell, J. A. R., et al. 2001, MNRAS, 321,239

Baranne, A., Mayor, M., \& Poncet, J.-L. 1979, Vistas Astron., 23, 279

Baranne, A., Queloz, D., Mayor, M., et al. 1996, A\&AS, 119, 1

Benz, W., \& Mayor, M. 1984, A\&A, 138, 183

Breger, M. 2000, ASP Conf. Ser., 210, 3

Breger, M., Handler, G., Nather, R. E., et al. 1995, A\&A, 297, 473

Breger, M., \& Bischof, K. M. 2002, A\&A, 385, 537

Burki, G., Cramer, N., Nicolet, B., et al. 2002, http://obswww. unige.ch/gcpd/ph13.html

Burnet, M., \& Rufener, F. 1979, A\&A, 74, 54

Dziembowski, W. A., \& Pamyatnykh, A. A. 1991, A\&A, 248, L11

Dziembowski, W. A. 1982, Acta Astron., 32, 147

ESA 1997, The HiPparcos and Tycho Catalogues, ESA SP-1200

Fitch, W. S. 1981, ApJ, 249, 218

Golay, M. 1980, Vistas Astron., 24, 141

Koen, C. 2001, MNRAS, 321, 44

Kurtz, D. W. 1978, ApJ, 221, 869

Kurtz, D. W. 1989, MNRAS, 238, 1077

Kurtz, D. W. 2000, ASP Conf. Ser., 210, 287

Labs, D., \& Neckel, H. 1970, Sol. Phys., 15, 79

Lomb, N. R. 1976, Astrophys. \& Space Sci., 39, 447

Lucy, L. B., \& Sweeney, M. A. 1971, AJ, 76, 544

Lucke, P. B. 1978, A\&A, 64, 367

Meylan, G., \& Hauck, B. 1981, A\&AS, 46, 281

Queloz, D., Allain, S., Mermilliod, J.-C., et al. 1998, A\&A, 335, 183

Rufener, F. 1964, Publ. Obs. Genève, A, 66, 413

Rufener, F. 1985, in Calibration of Fundamental Stellar Quantities, ed. D. S. Hayes, et al. (Reidel Publ. Co., Dordrecht), IAU Symp., 111, 253

Rufener, F. 1988, Geneva Photometric Catalogue, 4th ed., Obs. Genève

Scargle, J. D. 1982, ApJ, 263, 835

Schaller, G., Schaerer, D., Meynet, G., \& Maeder, A. 1992, A\&A, 96, 269

Turcotte, S., Richer, J., Michaud, G., \& Christensen-Dalsgaard, J. 2000, A\&A, 360, 603

Udry, S., Mayor, M., \& Queloz, D. 1999, ASP Conf. Ser., 185, IAU Coll., 170, 367 